\title{
BUSCANDO EL ANTÍDOTO NAT URAL EN LA LUCHA CONTRA EL "CARRIZO GIGANTE"
}

\section{E. Cortés ${ }^{1}$, M.A. Marcos ${ }^{1}$ y J. Goolsby ${ }^{2}$}

i Centro Iberoamericano de la Biodiversidad (CiBiO), Unidad Asociada al CSiC, ipab. Universidad de Alicante, España. ELENACORTESMENDOZA@HOTMAIL.COM MARCOS@UA.ES

2 United States Department of Agriculture, Agricultural Research Service. i 3 East Highway 83, Weslaco, TeXas, USA 78596 JOHN.GOOLSBy@ARs.USDA.gOV

Arundo donax. Linnaeus, 1753 también conocida como "carrizo gigante" es una planta invasora en muchas regiones subtropicales, tropicales y templadas de todo el mundo. Esta gramínea de gran tamaño (hasta 8 metros de altura), nativa del Viejo Mundo (desde la Península Ibérica hasta el sur de Asia) donde se cultivó durante miles de años con fines medicinales, hortícolas y culturales, fue introducida en Norte América, probablemente a principios del 1500 con la llegada de los primeros colonizadores españoles. En la actualidad, invade de manera muy agresiva ecosistemas ribereńos, principalmente del Suroeste de EEUU y del Norte de México. El hecho de que el área de California presente un clima Mediterráneo muy similar al del Sureste español, ha favorecido la rápida adaptación de esta maleza.

Las áreas ribereñas son zonas relativamente vulnerables a la invasión de especies vegetales exóticas tales como $A$. donax L. debido a la perturbación recurrente ocasionada por el transporte de propá- gulos vegetativos a través del cauce del rio y por la disponibilidad de agua para el crecimiento de la planta (HOOD \& NAIMAN, 2000; DECRUYENAERE \& HOLT, 2005).

Destaca la gran capacidad invasiva de esta gramínea a lo largo de distintos ríos californianos. Un ejemplo ilustrativo de esta expansión puede apreciarse a lo largo de Rio Grande (conocido como Rio Bravo en tierras mexicanas). Este rio ha marcado desde 1848 la frontera entre México y EEUU, y el carrizo gigante ha ocupado ya más de $600 \mathrm{Km}$ del mismo, favorecido en gran medida, por su capacidad de reproducción asexual mediante los fragmentos de rizomas y tallos que pueden moverse hasta nuevas zonas rio abajo.

Entre las principales consecuencias que trae consigo la presencia masiva de $A$. donax L. en Rio Grande destacan la erosión de las orillas alterando la morfología de los cauces, la competencia por los recursos hídricos en regiones áridas donde dichos recursos son críticos para el medio ambiente y la agri- 


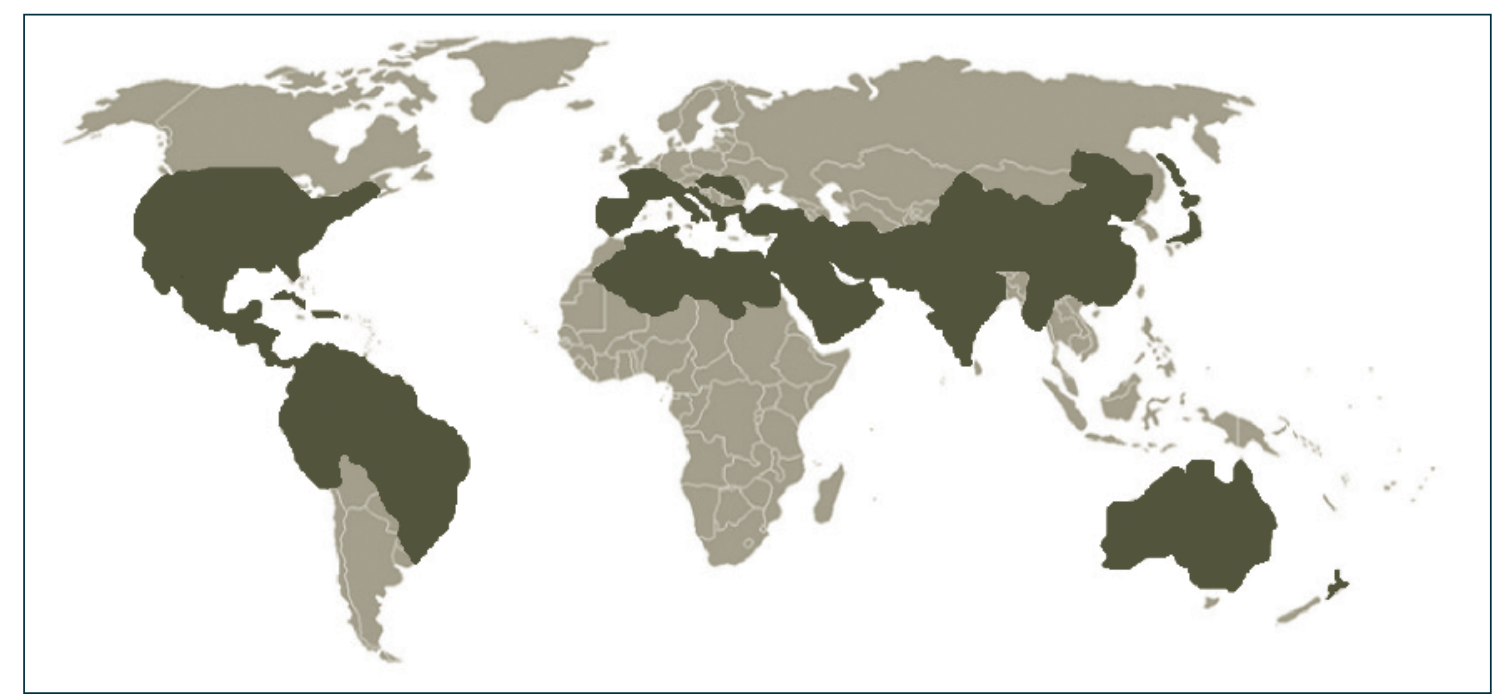

Figura 1. Distribución mundial de $A$. donax L.

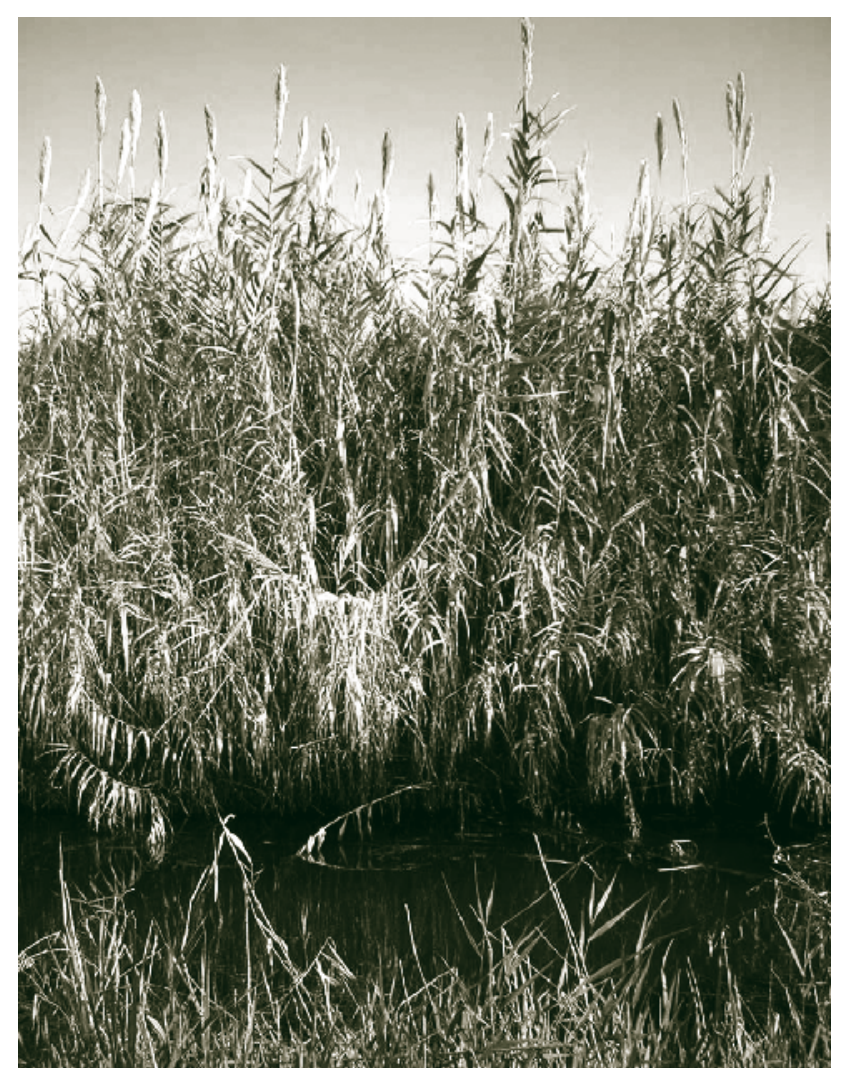

Figura 2. Imagen de una población en la Marjal PegoOliva (Límite entre las provincias de Alicante y Valencia). (E. Cortés, CIBIO).

cultura; y finalmente las consecuencias de pérdida de biodiversidad son especialmente dramáticas.

En los ríos en los que se extiende el carrizo formando grandes agrupaciones, logra desplazar a especies vegetales y animales autóctonas destruyendo los hábitat y modificando las características físico-químicas del lugar (BELL, 1997; DUDLEY, 2000; FRANDSEN, 1997; DECRUYENAERE \& HOLT, 2005).

Un ejemplo especialmente llamativo de este problema lo encontramos en el Valle de Cuatro Ciénegas (Coahuila, México); este paraje es considerado uno de los humedales de agua dulce más peculiares de la región Neártica, excepcionalmente biodiverso y con un alto grado de endemismos comparable al de las Islas Galápagos.

Aquí, en tan solo dos décadas el control de A. donax $\mathrm{L}$. se ha convertido en una prioridad, pues su proliferación amenaza la existencia de un importante conjunto de estromatolitos que se hallan en algunas de las pozas del valle debido a la alteración en las concentraciones de oxígeno y nutrientes que produce la presencia del carrizo gigante en este rio (GARCIA-PICHEL, 2004). La conservación de estas formas ancestrales de vida es de vital importancia para comprender el origen y evolución de la vida en nuestro planeta. A esto hay que sumar la extinción de Etheostoma segrex (HENDRICKSON et al, 2005) una especie de pez endémica del Rio Nadadores (Coahuila, México) muy próximo al valle de Cuatro Ciénegas como consecuencia de la imparable expansión de esta gramínea.

Diversas son las estrategias de manejo aplicadas sobre Arundo sp.; desde los de carácter mecánico 


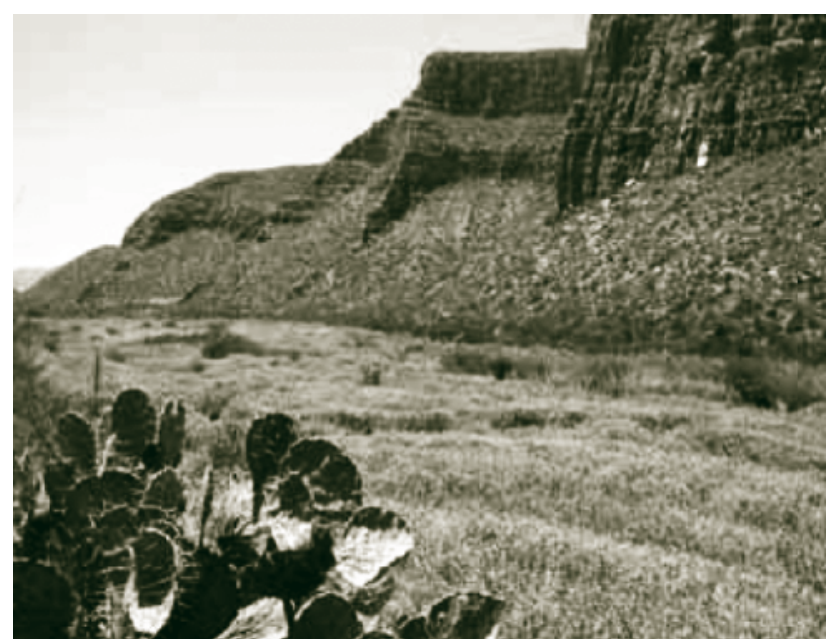

Figura 3. Río Nadadores próximo a Cuatro Ciénegas invadido por A. donax L. (J. Goolsby, USDA-ARS).

hasta los de tipo químico, resultando ambas inefectivas. Respecto a las primeras, se ha empleado entre otras, la realización de quemas con las que tan solo se elimina la parte aérea de la planta pero parte de los rizomas pueden permanecer intactos bajo tierra y rebrotar originando nuevas cañas solo una semana después de la quema, alcanzando ya para entonces incluso un metro de altura. Otros métodos como las podas también han resultado no ser exitosos y la completa extracción del terreno, es muy costosa.

Por otro lado, están los métodos químicos, el herbicida más empleado, el glifosato, requiere continuas aplicaciones, por lo que su aplicación en áreas como Rio Grande donde hablamos de cientos de kilómetros invadidos por esta maleza, resulta inviable y ambientalmente, poco conveniente.

Es por todo esto, que el control biológico se presenta como una alternativa de manejo a considerar seriamente para el control de las poblaciones de $A$. donax a largo plazo. Con este motivo y siendo conscientes de todo el problema medioambiental que genera el manejo de esta especie en Norte América, desde el USDA (Department of Agriculture of United States) se están llevando a cabo grandes esfuerzos por encontrar el método más eficaz y compatible con el medio ambiente para el control de esta maleza. Entre las diversas estrategias que se conocen de control biológico, se ha centrando el interés en la búsqueda de insectos procedentes del área de origen de Arundo donax L. que actúen como agentes de control biológico. No es la primera vez que donde se encuentra el origen del problema también se halla la solución al mismo.

En este marco nació el proyecto "Estudio de la biología de la cochinilla del Arundo en su área de origen" a partir del convenio establecido entre el CIBIO, Universidad de Alicante y el USDA y cuyo trabajo se desarrolla en el contexto de una Tesis Doctoral donde el principal reto es aportar datos científicos suficientes de la biología del pequeño insecto diaspídido, Rhizaspidiotus donacis (Leonardi, 1920), para su utilización como agente de control de A. donax L.

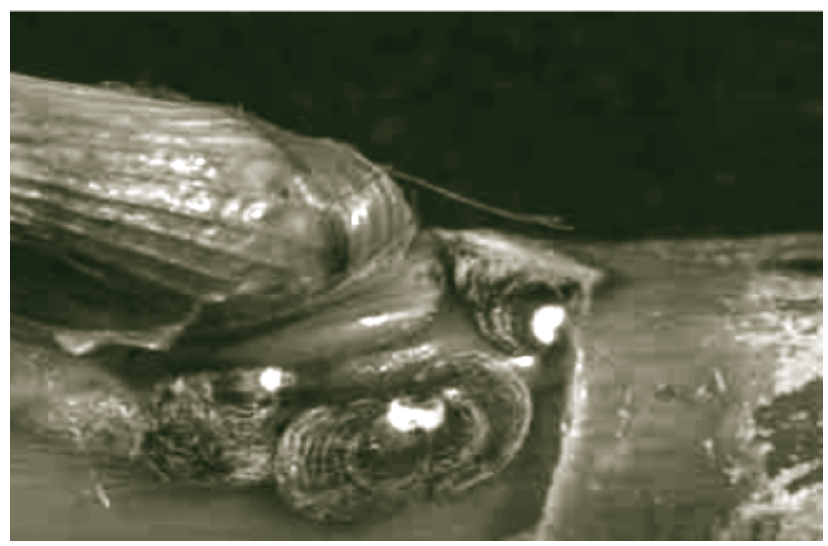

Figura 4.Imagen de Rhizaspidiotus donacis sobre Arundo donax L. (J. Goolsby, USDA-ARS).

Este insecto de no más de $1,5 \mathrm{~mm}$, que es uno de los posibles candidatos como agentes de control. Durante todo su ciclo biológico, se alimenta exclusivamente del carrizo gigante, situándose en la base de los brotes axilares y en el rizoma de la planta. Mediante su acción fitófaga, absorbe la savia del vegetal y consigue secarlo completamente poco a poco. Su acción se hace evidente cuando las cañas infectadas amarillean, el diámetro de su tallo principal disminuye haciéndolas más quebradizas y decrece producción de nuevas hojas y brotes laterales.

El impacto que se produce sobre la cańa se maximiza cuando a la acción de $R$.donacis se le suma la de un pequeño himenóptero, Tetramesa romana que también ha sido considerado por el USDA como candidato en la lucha contra Arundo donax L. ya que sus larvas se alimentan de los tallos y los brotes de esta caña. 

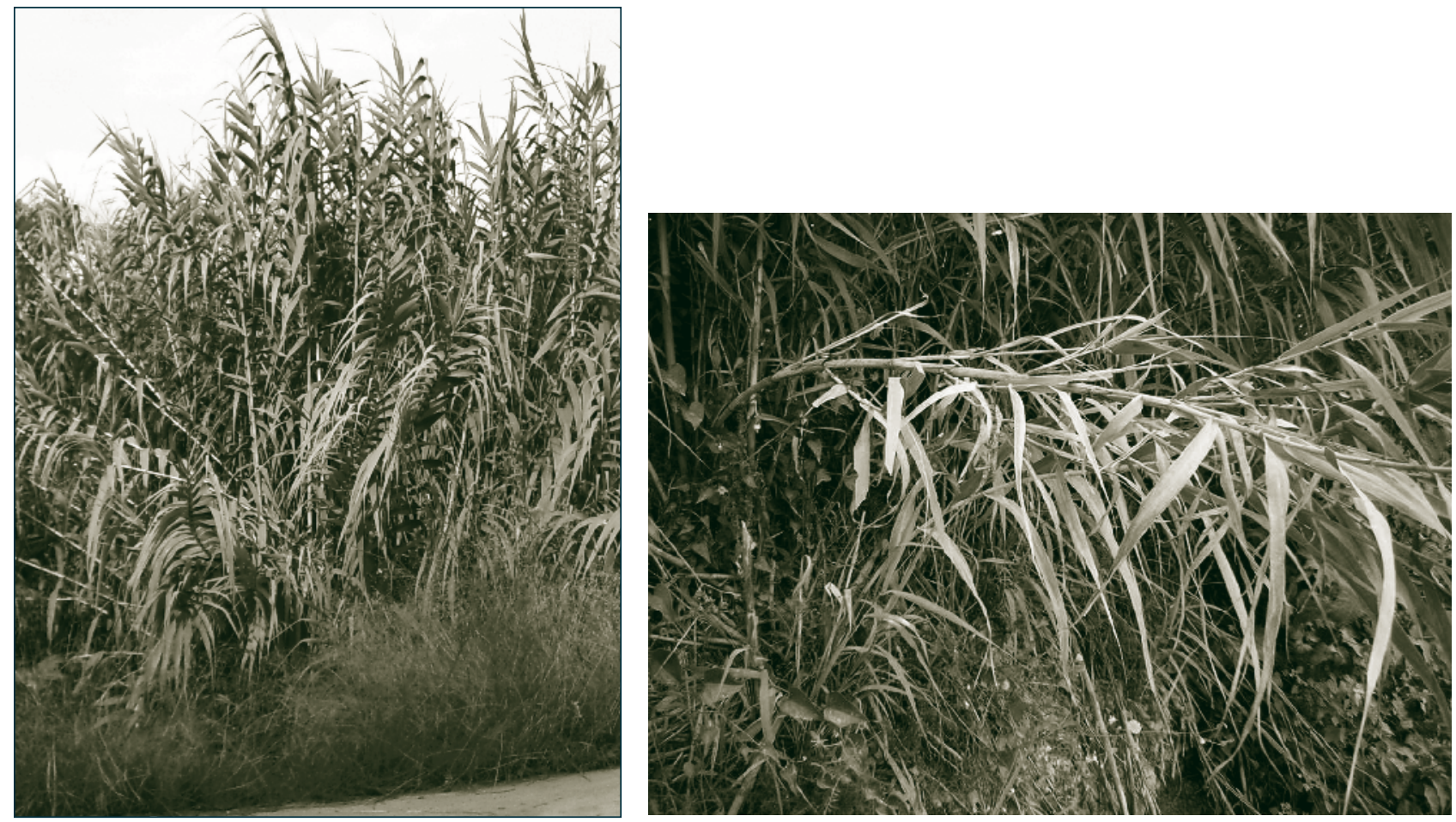

Figura 5. A la izquierda grupo de cañas sanas, a la derecha caña con síntomas evidentes de infección por $R$. donacis. (E Cortés, CIBIO).

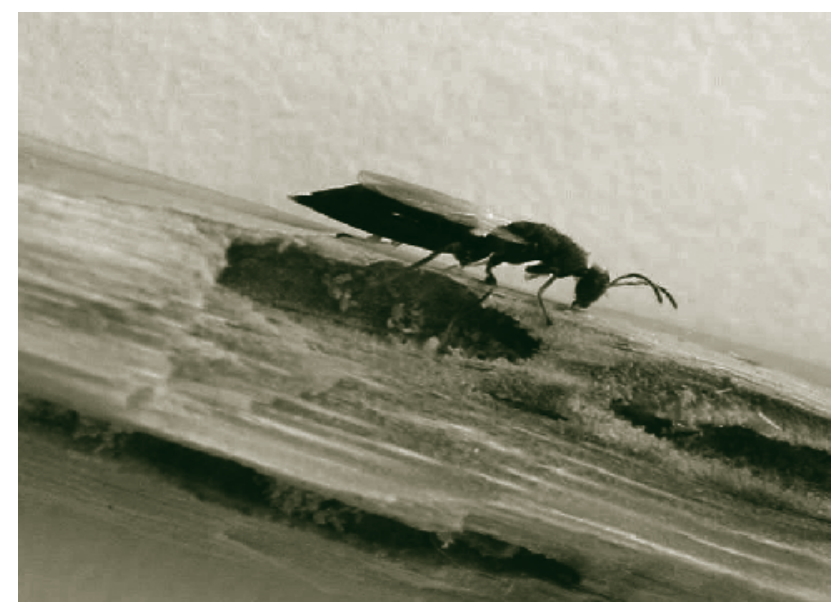

Figura 6. Imagen de un adulto de Tetramesa romana (J. Goolsby, USDA-ARS).

No obstante, estas liberaciones a lo largo del Rio Grande, deben hacerse con las suficientes garantías de que no van a provocar efectos secundarios indeseados y para ello, deben realizarse siguiendo una serie de protocolos, controles rigurosos y test necesarios para lograr que los gobiernos de Canadá, México y los EEUU acepten dichas sueltas, siendo imprescindible el visto bueno de todos los países implicados para poder ejecutarlas.
A partir de colectas procedentes principalmente del Sureste de España y de otras regiones mediterráneas cercanas a su área nativa, enviadas hasta las instalaciones del USDA, se realizan diferentes test de especificidad del huésped y estudios de eficacia pre-suelta dentro de las cuarentenas que ayuden a garantizar la especificidad, a determinar la longevidad, fecundidad y el tiempo de desarrollo de los agentes considerados, para poder asignar prioridades entre dichos agentes.

Frente a la expectativa depositada sobre estos candidatos, parece que los esfuerzos realizados en el marco de este proyecto comienzan a dar sus frutos, pues el pasado mes de Agosto, ya fue recomendada por los tres países anteriormente citados, la suelta de Tetramesa romana encontrándose actualmente en revisión la solicitud para Rhizaspidiotus donacis.

A día de hoy, estos pequeños insectos constituyen una gran esperanza en la lucha contra esta planta invasora, una esperanza por la que se seguirá trabajando desde el USDA y el CIBIO en pro de la conservación de la biodiversidad. 


\section{REFERENCIAS}

BELL, G. 1997. Ecology and management of Arundo donax, and approaches to riparian habitat restoration in Southern California. Plant Invasions: Studies from North America and Europe. 103-113.

DECRUYENAERE,J.G; HOLT, J.S. 2005.Ramet demography of a clonal invader, Arundo donax (Poaceae), in Southern California. Plant and soil. 277: 41-52.

DUDLEY, T.L. 2000. Arundo donax.L. Invasive Plants of California's Wildlands. 53-58.

FRANDSEN, P. 1997. Interagency cooperation to control giant cane (Arundo donax). Assessment and Management of Plant Invasions. 244-248.
GARCIA-PICHEL,F.A., AL-HORANI,J.D., FARMER, R. \& B.D. LUDWING. 2004. Balance between microbial calcification and metazoan bioerosion in modern stromatolitic oncolites. Geobiology. 2:1-49.

HENDRICKSON, D.A., GARCÍA DE LEÓN, F. \& SOUZA. V. 2005. Proceedings of the first meeting of Cuatro Ciénegas researchers.

HOOD, W.G. \& NAIMAN, R.J. 2000. Vulnerability of riparian zones to invasion by exotic vascular plants. Plant Ecol. 148:105-114. 